\title{
Erratum to: Metrics for measuring the consistencies of requirements with objectives and constraints
}

\author{
JungWon Byun · SungYul Rhew · ManSoo Hwang • \\ Vijayan Sugumaran $\cdot$ SooYong Park • \\ SooJin Park
}

Published online: 6 December 2013

(C) Springer-Verlag London 2013

\section{Erratum to: Requirements Eng \\ DOI 10.1007/s00766-013-0180-9}

Unfortunately, in the original publication, the fourth author's name (Vijayan Sugumara) and affiliation of all authors have been published incorrectly. The acknowledgment section was also missed.

The correct name of the fourth author is Vijayan Sugumaran. The correct affiliation of all authors and the acknowledgment section are given below.

The online version of the original article can be found under doi:10.1007/s00766-013-0180-9.

J. Byun $(\square) \cdot$ S. Rhew

Department of Computer Science and Engineering,

Soongsil University, Seoul, South Korea

e-mail: jimi01@ssu.ac.kr

S. Rhew

e-mail: syrhew@ssu.ac.kr

M. Hwang

Division of Computer Information, Shinheung College,

Seoul, South Korea

e-mail: mshwang@shc.ac.kr

\section{Sugumaran}

School of Business Administration, Oakland University,

Rochester, MI 48309, USA

e-mail: sugumara@oakland.edu

S. Park

Department of Computer Science and Engineering,

Sogang University, Seoul, South Korea

e-mail: sypark@sogang.ac.kr

\section{S. Park}

Graduate School of Management of Technology (MOT),

Sogang University, Seoul, South Korea

e-mail: psjdream@sogang.ac.kr

\begin{abstract}
Acknowledgments This research was supported by Next-Generation Information Computing Development Program through the National Research Foundation of Korea (NRF) funded by the Ministry of Science, ICT \& Future Planning (2012M3C4A7033348).
\end{abstract}

